\title{
The predictive role of cardiac fibrosis markers in progression of coronary heart disease associated with cardiac arrhythmias and arterial hypertension
}

\begin{abstract}
The markers of cardiac fibrosis were studied in patients with coronary heart disease (CHD), arterial hypertension $(\mathrm{AH})$ and atrial fibrillation $(\mathrm{AF})$, as well as with $\mathrm{CHD}$ and $\mathrm{AF}$ without $\mathrm{AH}$ and in the group with isolated CHD. The change of levels of serum fibrosis markers were found in patients with coronary heart disease: C-terminal propeptide of procollagen I type (PICP), matrix metalloproteinase-9 (MMP-9), tissue inhibitor of metalloproteinase-1 (TIMP-1); the highest level was combined with hypertension and AF. The risk of CHD increases with increasing of concentrations of MMP-9 and PICP and decreasing of concentration of TIMP-1. Most predictive has MMP-9. The risk of progression of coronary artery disease increases when the level of MMP-9 more than $27.25 \mathrm{ng} / \mathrm{ml}$, the level of PICP more than $99.6 \mathrm{ng} / \mathrm{ml}$, the level of TIMP-1 less than $465.3 \mathrm{ng} / \mathrm{ml}$. The risk of a critical stenosis of the coronary arteries increases with the level of MMP-9 more than 101.8ng/ $\mathrm{ml}$, more values PICP 195.6ng $/ \mathrm{ml}$. The odds ratio for the MMP-9 was 3.41 (CI 1,38-8,47, $\mathrm{P}=0.007$ ). The odds ratio for the PICP more than $195.6 \mathrm{ng} / \mathrm{ml}$ was 4.66 (confidence interval $1,68-12,9 ; \mathrm{p}=0.003$ )
\end{abstract}

Keywords: coronary heart disease, arterial hypertension, atrial fibrillation, matrix metalloproteinase - 9, tissue inhibitor metalloproteinase-1, carboxyterminal propeptid procollagen type I, prediction

\author{
Volume 3 Issue 2 - 2018
}

\section{Liubov Vasilez,' Olga Khlynova,' Natalia \\ Grigoriadi,' Aleksandr Tuev,' Ludmila Nekrutenko,' Vera Vustina,' Ekaterina Shishkina, ${ }^{2}$ Ksenia Trenogina, ${ }^{2}$ Olga Parshakova ${ }^{2}$}

'Department of Hospital Therapy, Federal State Budgetary Educational Institution of Higher Education, Russia

${ }^{2}$ The State Budgetary Healthcare Establishment of the Perm Krai Clinical Cardiological Clinic, Russia

\begin{abstract}
Correspondence: Olga Khlynova, Department of Hospital Therapy, Federal State Budgetary Educational Institution of Higher Education "Perm State Medical University named after academician E.A.Wagner "of the Ministry of Health of the Russian Federation, 614990, Serpukhovskaya str. 6-130, Perm, Russia,Tel 9024783482, Email olgakhlynova@mail.ru
\end{abstract}

Received: June 20,2018 | Published: June 27, 2018
Abbreviations: $\mathrm{CHD}$, coronary heart disease; $\mathrm{AF}$, atrial fibrillation; $\mathrm{AH}$, arterial hypertension; PICP, C-terminal propeptide of procollagen; MMP-9, matrix metalloproteinase-9; TIMP-1, tissue inhibitor of metalloproteinase-1

\section{Introduction}

There are convincing experimental studies on the role of cardiac fibrosis in the initiation and progression of atherosclerosis, ischemic heart disease (IHD), arterial hypertension (AH) as well as heart arrhythmias, in particular atrial fibrillation (AF). ${ }^{1,2}$ Numerous biomarkers participating in various stages of the inflammatory cascade as well as in the process of myocardial fibrosis are associated with unfavorable cardiovascular outcomes..$^{3-5}$ At present, the question remains open: is fibrosis a consequence of cardiac pathology or one of its triggers?. ${ }^{6}$ The role of serum and tissue fibrosis markers in the pathogenesis of IHD, $\mathrm{AH}$ and $\mathrm{AF}$, their relationships with the structural-functional and electrical remodeling of myocardium, as well as the need for determining these markers in clinical practice to clarify disease severity and risk of progression remains unclear.

There are assumptions those in patients with IHD, an increase in MMP-9 leads to an increase in the degradation of elastin and a decrease in myocardium elasticity. A decrease in the activity of TIMP1 , in contrast, can lead to the formation and accumulation of immature and unstable collagen synthesis products. This hypothesis is supported by a meta-analysis of the risk of progression of IHD associated with an elevated MMP-9 level. ${ }^{7}$ The meta-analysis discusses the results of 26 studies, which included 12,776 cases, where it was assumed that an increase in the level of MMP-9 is associated with progression of IHD. ${ }^{8}$
It has also been shown that the activation of MMP-9 is associated with coronary artery restenosis following stent implantation. ${ }^{2,7,9}$ Elevated levels of MMP-9 are associated with severe atherosclerotic processes in coronary arteries. ${ }^{7,10}$ Thus, metalloproteinases MMP-9, PICP and TIMP-1 may participate in the pathogenesis of IHD and AH, although the cause-and-effect relationship remains to be clarified. Currently, local and foreign literature is limited, hence the relevance of this study.

\section{Objective}

To study the markers of cardiac fibrosis in comorbid patients with ischemic heart disease, arterial hypertension and atrial fibrillation, and also to estimate the possible prognostic value of these parameters in the progression of coronary heart disease.

\section{Materials and methods}

The study included 108 patients (aged from 24 up to 45 years), who underwent inspection in the Perm regional hospital. 49 people had a combination of IHD, AH and AF, 36 of the patients had IHD without heart arrhythmia, and 23 patients had IHD with AF. The groups are comparable in gender distribution (Fisher's criterion, $\mathrm{p}=0.51$ ) and age (U-test, $\mathrm{p}=0.11$ ).

All patients underwent general clinical examination, echocardiography, coronary angiography, and determination of serum markers of cardiac fibrosis, including matrix metalloproteinase (MMP-9), tissue inhibitor metalloproteinases-1 (TIMP-1) and C-terminal propeptide of procollagen type I (PICP) in blood serum. Blood sampling was carried out under sterile conditions according to standard methods. The blood samples were immediately centrifuged, 
the plasma was frozen at $-20^{\circ} \mathrm{C}$. Storage of plasma at a temperature regime of $-20-70^{\circ} \mathrm{C}$ lasted no more than 6 months. The levels of MMP-9, TIMP-1 and PICP in the blood were measured using enzyme immunoassay test systems. The PICP was determined using the Metra CIPC EIA Kit (Quidel Corporation, USA) diagnostic kit using enzyme immunoassay. To assess the level of MMP-9, a set of reagents "Human MMP-9 ELISA" (Bender MedSystems, Austria) were used The level of TIMP-1 was determined using the Human TIMP-1 ELISA reagent kit (Bender MedSystems, Austria).

The research has been approved by local ethical committee (the minutes no 8 2/12/2016). All patients have signed voluntary consent to participation in a research and to their division according to groups of observation.

\section{Statistical analysis}

The results were processed statistically using the computer program Statistical 10.1. The design of the study is presented by an open controlled trial.

\section{Results and discussion}

The markers of fibrosis and inflammation were studied in patients with IHD in combination with $\mathrm{AH}$ and AF, IHD and AF without $\mathrm{AH}$, as well as in patients with isolated IHD and practically healthy individuals.

When analyzing the values of MMP-9, it was found that this marker significantly differs from the comparison group in patients with ischemic heart disease. Thus, in groups with the combination of IHD, $\mathrm{AH}$ and $\mathrm{AF}$, as well as IHD with $\mathrm{AF}$ without hypertension, its values were $115.6 \mathrm{ng} / \mathrm{ml}(\mathrm{p}=0.0001)$ and $106.4 \mathrm{ng} / \mathrm{ml}(\mathrm{p}=0.0003)$, respectively. In patients with isolated ischemic heart disease-107.6ng $/ \mathrm{ml}(\mathrm{p}=0.0003)$. The values of MMP-9 in the groups with IHD were maximal in patients with $\mathrm{AH}$ and $\mathrm{AF}$, slightly less in patients with isolated ischemic heart disease, as well as in IHD with AF. There were no significant differences between the groups. Thus, in the presence of IHD, a higher MMP-9 level is observed. Additional aggravating factors probably can be considered as the presence of AF and $\mathrm{AH}$ in these patients, when MMP-9 metabolic disorders are multiplied by comorbid status.

Table I Multiple regression results of the predictor value of inflammatory and fibrosis markers in the development of IHD

\begin{tabular}{llllll}
\hline Indicators & Beta & BETA art error & B & $\mathbf{t}$ & $\mathbf{p}$ \\
\hline Free member & & & 0,144 & 1,98 & 0,044 \\
MMP-9 & 0,796 & 0,037 & 0,008 & 21,53 & $<0,00000$ I \\
PICP & 0,075 & 0,033 & 0,0003 & 2,26 & 0,025 \\
TIMP-I & $-0,164$ & 0,035 & $-0,0005$ & $-4,566$ & 0,000009 \\
\hline
\end{tabular}

The multiple regression equation is as follows:

$$
Y=0,144+0,008 * X_{1}+0,0003 * X_{2}-0,0005 * X_{3}
$$

As: $\mathrm{Y}$ - dependent variable (the presence of IHD), 0.757 - constant index, $X_{1}$ - concentration of MMP-9 $(\mathrm{ng} / \mathrm{ml}), X_{2}$ - concentration of CICP (ng/ml), $X_{3}$ - concentration of TIMP-1ng/ml.

Thus, the analysis of multiple regressions indicated the values of MMP-9, PICP and TIMP as predictors for the development of IHD. At the same time, the risk of developing coronary artery disease increases with a rise in the concentration of MMP-9, PIsP, and a decrease in TIMP-1. Visual assessment of these indicators in patients with IHD and without it confirms the significance of the model (Figure 1).
When analyzing the values of the TIMP-1 indicator, a slightly different picture was obtained: the values of MMP-9 and TIMP-1 correlate in an inverse proportion, which is comparable with the few literature data available. The level of TIMP-1 in healthy subjects was $496.8 \mathrm{ng} / \mathrm{ml}$, the lowest values of TIMP-1 were obtained in patients with IHD. Thus, in patients with isolated ischemic heart disease and IHD with AF they were $404.4 \mathrm{ng} / \mathrm{ml}, \mathrm{p}=0.003$ and $406 \mathrm{ng} / \mathrm{ml}, \mathrm{p}=0.003$, respectively. The lowest level of TIMP-1 was observed in patients with a combination of IHD, AH and AF- 359.5ng/ml, $\mathrm{p}=0.0001$. Groups with IHD had significantly different TIMP-1 values: IHD, $\mathrm{AH}, \mathrm{AF}$ with the group of IHD and AF, $\mathrm{p}=0.0001$; IHD, AH, AF with isolated ischemic heart disease, $\mathrm{p}=0.001$.

By PICP level, all studied groups of patients also significantly differed from healthy ones. Its highest values were in patients with isolated ischemic heart disease and amounted to $176.4 \mathrm{ng} / \mathrm{ml}$, $\mathrm{p}<0.001$; the combination of IHD and AF-174.1ng/ml, $\mathrm{p}<0.001$; in patients with IHD, AH and AF- 163.2ng/ml, p<0.001 IHD. Thus, in the analysis of fibrosis markers, it was found that the values of MMP9 and PICP are maximal in the groups of patients with IHD both with isolated angina and in groups with the combination of IHD and AF. The presence of arrhythmia and hypertension worsens the actual indices. The level of TIMP-1 is inversely proportional to MMP-9 and procollagen. The data obtained represent significant imbalances in the system of metalloproteinases in favor of fibrotic transformations in the myocardium, progressing with an increase in comorbid status.

During statistical processing of the material, significant differences in the values of fibrosis markers were found depending on the presence of IHD. This served as a basis for assessing the predictor value of fibrosis markers in the risk of coronary artery stenosis. The construction of a model for the development of IHD was performed on the whole array of patients - 108 people. To analyze the relationship between possible predictors of IHD, the multiple regression method was used. Multivariate regression analysis included the following indicators: PICP, TIMP-1 and MMP-9. The R2 model determination coefficient amounted to 0.83 , which indicates the presence of a strong relationship between IHD and selected predictors (R2>0.75 strong). The F criterion is $212.1, \mathrm{p}<0.0000001$, which represents the significance of the constructed model. Significant predictor value was observed for PICP, TIMP-1 and MMP-9 values (Table 1).

For the possibility of practical use of these indicators as predictors of the development of IHD, an assessment of their diagnostic effectiveness was conducted and specific threshold values were determined. For this purpose, a ROC analysis was performed and the ROC curves were plotted (Figure 2). The statistically significant differences between AUC MMP-9 and AUC TIMP-1 ( $p=0.0001)$, as well as between AUC MMP-9 and AUC PICP $(p<0.0001)$ were revealed, which indicates a greater predictor significance of MMP9. When comparing AUC TIMP-1 and AUC PICP, no statistical significance was found, which represents an equal degree of their prognostic value (Table 2). 


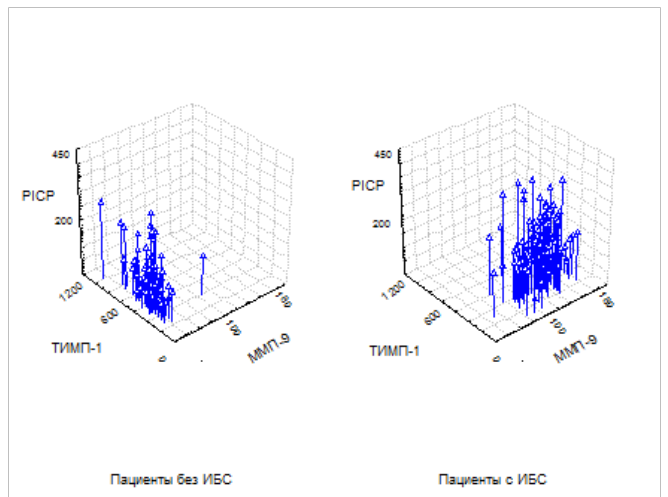

Figure I TIMP-I, MMP-9 and PICP values in patients with and without IHD.

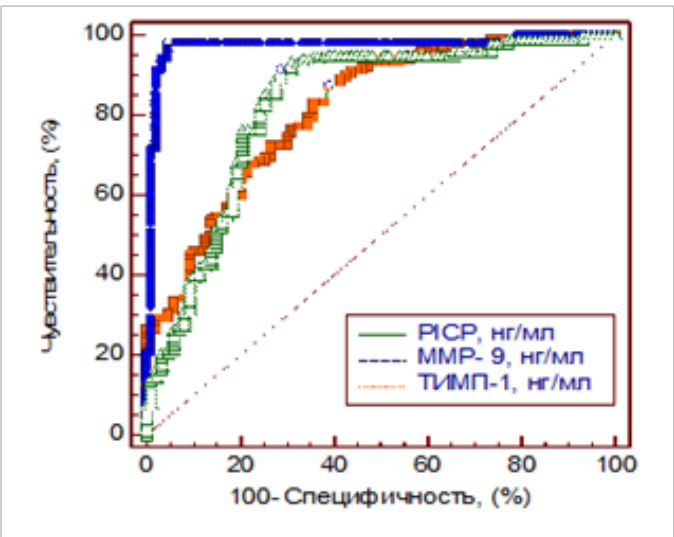

Figure 2 Comparative characteristics of MMP-9,TIMP-I, PICP ROC curves.

Table 2 ROC-curve characteristics for MMP-9, TIMP-I and PICP
To ensure the possibility of a practical application of the data obtained, threshold values of MMP-9, PICP and TIMP-1 were determined at which the balance of specificity and sensitivity of the indicator used is achieved. For all indicators, the Jouden index and the value associated with it were calculated (Table 3 ). The Jouden index for PICP was 0.41 (confidence interval 0.30-0.51), the associated value (separation point) of PICP greater than $99.6 \mathrm{ng} / \mathrm{ml}$ (sensitivity $87.5 \%$, specificity $53.5 \%$ ). The Jouden index for MMP-9 was 0.91 (confidence interval 0.85-0.96), the associated value (separation point) of MMP-9 greater than $27.25 \mathrm{~g} / \mathrm{ml}$ (sensitivity $98 \%$, specificity 93\%). The Jouden index for TIMP-1 was 0.62 (confidence interval $0.30-0.51$ ), the associated value (separation point) TIMP-1 less than $465.3 \mathrm{~g} / \mathrm{ml}$ (sensitivity $91.2 \%$, specificity $71.3 \%$ ).

Further study of these indicators was conducted with respect to their significance in terms of the development of significant stenosis $(>75 \%)$ in patients with IHD. The area of the ROC curve for MMP-9 was $0.662(p=0.0024)$, which indicates a good quality of the model (Figure 1). Jouden's index was 0.306 , and the associated criterion was more than $101.8 \mathrm{ng} / \mathrm{ml}$. The area of the ROC curve for TIMP-1 was $0.585(\mathrm{p}=0.141)$, and hence this parameter was excluded from the prognostic model. The area of the ROC curve for PICP was 0.680 $(\mathrm{p}=0.0007)$ (Figure 2). Jouden's index was 0.345 , and the associated criterion was more than $195.6 \mathrm{ng} / \mathrm{ml}$.

The odds ratio for MMP-9 of more than $101.8 \mathrm{ng} / \mathrm{ml}$ provided that the PICP value remains constant is 3.41 (confidence interval 1.38-8.47, $\mathrm{p}=0.007$ ). The odds ratio for PICP of more than 195.6ng/ $\mathrm{ml}$, provided that the value of MMP-9 remains constant, is 4.66 (confidence interval 1.68-12.9, $\mathrm{p}=0.003$ ).

\begin{tabular}{lll}
\hline Comparison of ROC curves & Difference between AUC & $\mathbf{p}$ \\
\hline MMP-9 ROC curve $\sim$ IMP-IROC curve & 0,140 & 0,000 I \\
MMP-9 ROC curve PICP ROC curve & 0,149 & $<0,000$ I \\
PICP ROC curve TIMP-IROC curve & 0,0009 & 0,86 \\
\hline
\end{tabular}

Table 3 Threshold values of MMP-9, PICP and TIMP-I

\begin{tabular}{llll}
\hline & MMP-9 & TIMP & PICP \\
\hline Jouden index & 0,91 & 0,62 & 0,41 \\
95\% Jouden index confidence interval & $0,85-0,96$ & $0,51-0,71$ & $0,30-0,51$ \\
Criteria associated with the Jouden index & $>27,25$ & $<465,3$ & $>99,6$ \\
$95 \%$ criteria confidence interval & $25,77-27,25$ & $425,2-484,4$ & $68,4-100$ \\
\hline
\end{tabular}

\section{Conclusion}

i. Ischemic heart disease is accompanied by an imbalance in the level of serum fibrosis markers: MMP-9, TIMP-1 and type I $\mathrm{C}$-terminal collagen propeptide. The maximum increase in MMP9 was observed in groups with $\mathrm{AF}$ and $\mathrm{AH}$, especially when combined with AH. Procollagen values are significantly higher in groups with arrhythmia, reaching a maximum in patients with IHD, AH and AF.

ii. The risk of developing IHD increases with an increase in the concentration of MMP-9 and PICP and a decrease in the concentration of TIMP-1. MMP-9 has a great predictor value. TIMP-1 and PICP have equal prognostic significance. The risk of progression of IHD increases with a level of MMP-9 greater than $27.25 \mathrm{ng} / \mathrm{ml}$, PICP values greater than $99.6 \mathrm{ng} / \mathrm{ml}$ and a TIMP-1 value less than $465.3 \mathrm{ng} / \mathrm{ml}$.

iii. The risk of developing critical stenosis of the coronary arteries increases with a MMP-9 level greater than $101.8 \mathrm{ng} / \mathrm{ml}$, PICP values greater than $195.6 \mathrm{ng} / \mathrm{ml}$. The odds ratio for MMP-9 is 3.41 (confidence interval 1.38-8.47, $\mathrm{p}=0.007$ ). The odds ratio for $\mathrm{PICP}$ is more than $195.6 \mathrm{ng} / \mathrm{ml}$ is 4.66 (confidence interval 1.68 $12.9, \mathrm{p}=0.003)$. 


\section{Acknowledgments}

None.

\section{Conflict of interest}

Author declares no conflict of interest.

\section{References}

1. Mora S, Musunuru K, Blumenthal RS. The clinical utility of highimplication of JUPITER on current practice guidelines. Clin Chem 2009;55(2):219-228.

2. Yousuf O, Mohanty BD, Martin SS, et al. High-sensitivity C-reactive protein and cardiovascular disease: a resolute belief or an elusive link? $J$ Am Coll Cardiol. 2013;62(5):397-408.

3. Ridker PM, Silvertown JD. Inflammation, C-reactive protein, and atherothrombosis. J Periodontol. 2008;79(8):1544-1551.

4. Tanus-Santos JE. Matrix metalloproteinases: a target in in-stent restenosis. Cardiology. 2013;124(1):49-50.

5. Василец Л.М, Агафонов АВ, Хлынова ОВ, et al. Прогнозирование фибрилляции предсердий по содержанию сывороточных маркеров воспаления при артериальной гипертензии. Казанский медицинский журнал. 2012;93(4):642-646
6. Koenig W. High-sensitivity C-reactive protein and atherosclerotic disease: from improved risk prediction to risk-guided therapy. Int $J$ Cardiol. 2013;34(15):5126-5134.

7. Balta S, Demirkol S, Celik T, et al. Active matrix metalloproteinase-9 is associated with clinical in-stent restenosis. Cardiology. 2013;125(2):86.

8. Opstad TB, Pettersen AÅ, Arnesen H, et. al. The co-existence of the IL-18+183 A/G and MMP-9 -1562 C/T polymorphisms is associated with clinical events in coronary artery Disease Patients. PLOS ONE. 2013;8(9):e74498.

9. Tarr GP, Williams MJ, Wilkins GT, et al. Intra-individual changes of active matrix metalloproteinase- 9 are associated with clinical in-stent restenosis of bare metal stents. Cardiology. 2013;124(1):28-35.

10. Tanindi A, Sahinarslan A, Elbeg S, et al. Relationship between MMP1, MMP-9, TIMP-1, IL-6 and Risk Factors, Clinical Presentation, Extent and Severity of Atherosclerotic Coronary Artery Disease. Open Cardiovasc Med J. 2011;5:110-116. 